Research Paper

\title{
Systemic Angiopoietin-1/2 Dysregulation in Pediatric Sepsis and Septic Shock
}

\author{
Elliot Melendez ${ }^{1 凶}$, Jane E. Whitney², Jackson S. Norton³, Melanie Silverman³, Susanna Harju-Baker4, \\ Carmen Mikacenic ${ }^{4}$, Mark M. Wurfel ${ }^{4}$, W. Conrad Liles ${ }^{4}$ \\ 1. Division of Pediatric Critical Care, John Hopkins All Children's Hospital, St. Petersburg, FL \\ 2. Division of Critical Care, Children's Hospital of Philadelphia, Philadelphia, PA \\ 3. Division of Medicine Critical Care, Boston Children's Hospital, Boston, MA \\ 4. Department of Medicine, University of Washington, Seattle, WA \\ $\square$ Corresponding author: Elliot Melendez, MD, Critical Care, Johns Hopkins All Children's Hospital, 501 6th Avenue South, St. Petersburg, FL 33701, \\ 727-787-3220. Email: emelend1@jhmi.edu \\ (c) Ivyspring International Publisher. This is an open access article distributed under the terms of the Creative Commons Attribution (CC BY-NC) license \\ (https://creativecommons.org/licenses/by-nc/4.0/). See http://ivyspring.com/terms for full terms and conditions.
}

Received: 2018.06.07; Accepted: 2018.10.31; Published: 2019.01.01

\begin{abstract}
Background: Angiopoietin- 1 and -2 are vascular growth factors that exert opposing effects on endothelial activation and dysfunction. The aim of this study was to assess the association of these biomarkers with outcomes in children with sepsis.

Methods: Biomarkers were assayed from the blood collected in an emergency department prior to any intervention. Predictor variables were Ang-1 and Ang-2 levels and the Ang-2/Ang-1 ratio. Outcomes included mortality, length of time on vasopressors, and ICU and hospital lengths of stay. The Pediatric RISk of Mortality III Score was calculated. A vasoactive inotrope score was calculated every 12 hours.

Results: Forty-five children with sepsis and 49 with septic shock were analyzed. The median Ang-2 was higher in septic shock. The Ang-2/Ang-1 ratio was approximately 2-fold greater in those with septic shock. The Ang-2/Ang-1 ratio was associated with higher doses of vasoactive agents at 12 hours and longer ICU length of stay. In septic shock, for every 0.35 unit increase in the Ang-2/Ang-1 ratio, the PRISM III score increased by 1 .

Conclusions: The Ang-2/Ang-1 ratio was higher in children with septic shock. Ang-2/Ang-1 was associated with higher vasoactive agents, longer ICU length of stay, and correlated with the severity of illness score.
\end{abstract}

Key words: Angiopoietin, Pediatric, Sepsis, Septic Shock, Outcome, Biomarker

\section{Introduction}

Septic shock remains a significant problem in the pediatric patient population and is associated with substantial morbidity and mortality (9.8\%).1,2 Endothelial activation/dysfunction, which causes increased vascular permeability, changes in systemic vascular resistance, and dysfunction of anti-coagulant factors, is a major cause of clinical deterioration in sepsis.1,3,4 Unfortunately, there are few individual biomarkers, or combination of biomarkers, that are useful for generalized clinical application to assess for endothelial dysfunction. ${ }^{5}$

Angiopoietin-1 and -2 (Ang-1 and Ang-2) are members of a family of vascular growth factors that play opposing physiological roles in endothelial activation and dysfunction. Both Ang-1 and Ang-2 bind to the same cognate receptor, the tyrosine kinase with immunoglobulin-like loop epidermal growth factor homology domain 2 (Tie-2). Ang-1 binding promotes vascular growth and stability, suppresses inflammation, and promotes endothelial cell survival.6-8 In contrast, Ang-2 binding promotes vascular activation, vascular inflammation, microvascular leak, and neoangiogenesis.6,9,10

Several studies in the adult intensive care unit (ICU) setting have shown that high levels of systemic Ang-2 in peripheral blood are associated with 
systemic inflammatory response and/or sepsis. Those individuals with higher levels of Ang-2 have an increased 28-day mortality, while higher levels of Ang-1 are associated with lower mortality.11,12 Similarly, for children with severe infection and/or sepsis, several studies have correlated angiopoietin levels with worse outcomes. ${ }^{13-16}$ However, all these studies involved plasma samples obtained after arrival to the ICU, and thus, angiopoietin levels could reflect both the patient's initial disease state and responses to therapeutic interventions, such as fluid and vasoactive agents administered. There are no published studies that have assessed the predictive/prognostic utility of circulating Ang-1 and Ang-2 levels obtained in pediatric patients at the time of emergency department (ED) presentation.

The primary purpose of this study was to determine whether children who present to the ED with severe sepsis and septic shock have elevations in circulating Ang-1 and Ang-2 as compared to children with sepsis without shock. Second, we sought to assess whether in patients with septic shock, the degree of biomarker elevation is associated with outcomes, specifically increased mortality, longer ICU and hospital length of stay (LOS), longer duration of vasoactive agent use, and increased organ dysfunction. Finally, because there is currently no ED severity of illness (SOI) score specific to pediatric septic shock, we aimed to determine if Ang-1 and/or Ang-2 concentration correlates with later ICU SOI scores. We hypothesized that a higher Ang-2 and lower Ang-1 levels, and that a higher Ang-2/Ang-1 ratio would be associated with worse outcomes in pediatric severe sepsis and shock.

\section{Methods}

This study was a retrospective study of children who presented to the ED of Boston Children's Hospital (Boston, MA) meeting the consensus definitions for sepsis and septic shock. ${ }^{17}$ Boston Children's Hospital is a pediatric tertiary care hospital with an ED volume of $>59,000$ visits per year.

From October 1, 2012 to April 30, 2014, children 0-21 years of age meeting diagnostic criteria for septic shock admitted to an intensive care unit were enrolled in a study of biomarkers in sepsis. An automated log of all ED admissions in the preceding 24hrs was reviewed to assess if any patient who was admitted from the ED met criteria for septic shock. The definitions of sepsis and septic shock was determined in accordance by the criteria set forth by the International Pediatric Consensus Conference. ${ }^{17}$ For every child with septic shock enrolled, a child on the daily ED admission log who met a definition for sepsis without shock or organ dysfunction, ${ }^{17}$ but not admitted to the intensive care unit, was also enrolled. Selection was random, selecting the next available patient with criteria for sepsis, and matching was not performed. Patients were only included if their ED blood sample was obtained prior to the administration of antibiotics and initiation of vasoactive agents, and if intravenous fluid boluses were given, if the volume given was less than 40 $\mathrm{mL} / \mathrm{kg}$. To account for expected patient dropout due to insufficient volume of plasma being left over after routine analysis, additional enrollment was permitted with a goal to maintain 1:1 enrollment.

Patients were excluded if there was pre-existing severe renal disease with creatinine clearance less than $45 \mathrm{~mL} / \mathrm{min}$ documented in the electronic health record (EHR), pre-existing cardiac disease or previously described ventricular dysfunction, diagnosis of severe sepsis or septic shock for $>24$ hours if referred from an outside facility, or if labs were not sent from the emergency department. If a patient had congenital heart disease that was repaired to normal anatomy and normal function was documented on the last echocardiogram, they were eligible for enrollment; but if this information was not available, they were excluded. Patients who had received $>40 \mathrm{~mL} / \mathrm{kg}$ of fluid prior to arrival were excluded. Patients who were found to have insufficient left-over plasma volume to undergo biomarker analysis were excluded. Informed consent to participate in this study was obtained from a parent or legal guardian, and assent obtained from the patient when possible within 24 hours of identification and up to 5 days post presentation, based on parent availability. The Institutional Review Board at Boston Children's Hospital approved this study.

Data related to clinical presentation and hospital course was abstracted from the EHR, including patient demographics, pre-existing conditions, laboratory and radiographic results, and outcomes, such as mortality, length of time on vasoactive agents, ICU LOS, and hospital LOS. Source of infection was recorded and confirmed if a pathogen grew in cultures of urine, blood, or stool, or by viral testing not considered to be a contaminant per accepted diagnostic criteria. Pneumonia was diagnosed if chest radiograph was interpreted by a radiologist as pneumonia. Skin/soft tissue infection was confirmed if a pathogen was identified from abscess culture or by clinical diagnosis as detailed in the EHR. Culture negative sepsis was recorded when no identified source of infection was evident on review of the EHR. SOI scores were calculated for patients admitted to the ICU including the Pediatric RISk of Mortality III (PRISM III) score $^{18}$ at 12 hours after ICU admission, 
and the Pediatric Logistic Organ Dysfunction (PELOD) score, ${ }^{19}$ calculated every 24 hours for 72 hours, beginning from the time of ICU admission. For patients requiring vasoactive agents, the duration of treatment was computed. A vasoactive inotrope score (VIS) was calculated in 12 hour intervals to summarize the number, dose, potency and duration of vasoactive agents required according to the following formula: vasoactive inotrope score $=$ (dopamine dose $\times 1)+($ dobutamine dose $\times 1)+$ (epinephrine dose $x$ 100) + (norepinephrine dose $x$ $100)+($ milrinone dose $\times 10)+($ vasopressin dose $x$ 10,0000). ${ }^{20}$ We assessed the maximum vasoactive inotrope score in the 12-hour period to capture scenarios where rapid weaning of vasoactive agents may have occurred. As vasoactive agents are recorded clinically each hour and with changes, we calculated the VIS for each hour in the 12-hour period, and selected the maximum score. ${ }^{20}$ All clinical data were extracted from the EHR prior to biomarker analysis.

As the patient plasma samples had been used in a prior analysis, patients were only included in the study if there was residual plasma remaining to permit biomarker analysis, and thus represent a convenience sample. Ang-1 and Ang-2 were assayed only from the first blood samples collected prior to any ED interventions. Collected plasma was maintain frozen at $-80^{\circ} \mathrm{C}$, thawed once for the prior analyses, and re-frozen until the time of Angiopoietin analysis. Plasma concentrations of Ang-1 and Ang-2 were measured at the same time using a multiplex immunoassay (Meso Scale Discovery, Rockville, MD). Samples were diluted to fit within the dynamic range of the assay: Ang-1 (3 pg/mL to $100,000 \mathrm{pg} / \mathrm{mL}$ ); and Ang-2 $(0.5 \mathrm{pg} / \mathrm{mL}$ to $10,000 \mathrm{pg} / \mathrm{mL}$. Samples below the lower limit of detection or above the upper limit of detection were assigned those values, respectively. Results of the assays were not available to the physician(s) caring for the patient(s).

A quantile (median) regression model was estimated with the biomarker as the outcome and the patient group (sepsis vs. septic shock), along with any demographic or clinical covariates, as predictors. Given that Angiopoietin levels were measured in $\mathrm{pg} / \mathrm{mL}$ and ranged from 2,052 to 241,758 , regression coefficients for this variable may be so small as to obscure clinical interpretability. To enhance interpretability, Angiopoietin was re-scaled by collapsing the score into deciles, such that the resulting coefficients were interpreted as the percent change in the clinical outcome corresponding to a ten-percentile increase in Angiopoietin. Any demographic or clinical factors associated with a patient group at the $p<0.05$ level were considered a potential confounder and included in any subsequent multivariable analyses. In those with septic shock, a quantile regression model was estimated also for outcomes with the biomarker level as the predictor. Chi square tests were used for categorical variables. Because of skewness, nonparametric statistical analyses were used and included the Mann-Whitney $\mathrm{U}$ test, Wilcoxon rank sum test, and Kruskal-Wallis method, as appropriate. Analyses were not adjusted for multiple comparisons. Study data were collected and managed using the Research Electronic Data Capture (REDCap) tool. ${ }^{21}$ Data were analyzed using STATA 13 (StataCorp. Stata Statistical Software: Release 13. In. College Station, TX: StataCorp LP; 2013.

\section{Results}

A total of 119 children with sepsis (without septic shock) and septic shock had been enrolled in the original study, 61 with sepsis and 58 with septic shock. Forty-five children with sepsis (without septic shock) and 49 children with septic shock has sufficient residual plasma available for biomarker analysis. Sepsis cases were older in years than septic shock cases [4.0 (IQR 1.8, 11.0) vs. 12.2 (IQR 8.1, 16.3), p= 0.001]. There was no difference in gender, temperature, initial white blood count, source of infection, or in underlying medical condition between groups (Table 1). None of the patients enrolled received fluid boluses, antibiotics, or vasoactive agents prior to the initial sample being acquired in the ED.

Table 1: Demographics and clinical characteristics

\begin{tabular}{|c|c|c|c|}
\hline Demographics & Sepsis $(n=45)$ & Septic Shock $(n=49)$ & p-value \\
\hline Male (n, \%) & $21(56.8)$ & $16(43.2)$ & 0.165 \\
\hline $\begin{array}{l}\text { Age in years (Median, } \\
\text { IQR) }\end{array}$ & $4.0(1.8-11)$ & $12.2(8.1-16.3)$ & 0.001 \\
\hline $\begin{array}{l}\text { Triage Temp (Median, } \\
\text { IQR) }\end{array}$ & $38.4(37.8-38.9)$ & $38(37.2-38.9)$ & 0.111 \\
\hline WBC (Median, IQR) & $11.3(5.0-18.4)$ & $11.0(6.8-18.9)$ & 0.608 \\
\hline \multicolumn{4}{|l|}{ Etiology of Infection } \\
\hline Culture Negative Sepsis & $11(24.4 \%)$ & $12(24.5 \%)$ & 0.996 \\
\hline Viral & $11(24.4 \%)$ & $5(10.2 \%)$ & 0.066 \\
\hline Skin/Soft Tissue & $6(13.3 \%)$ & $3(6.1 \%)$ & 0.235 \\
\hline Pneumonia & $6(13.3 \%)$ & $9(18.4 \%)$ & 0.506 \\
\hline Urine & $5(11.1 \%)$ & $4(8.2 \%)$ & 0.733 \\
\hline Bacteremia & $3(6.7 \%)$ & $7(14.3 \%)$ & 0.321 \\
\hline Gastrointestinal & $3(6.7 \%)$ & $5(10.2 \%)$ & 0.716 \\
\hline Other & $0(0 \%)$ & $4(8.2 \%)$ & 0.118 \\
\hline \multicolumn{4}{|l|}{ Comorbidity } \\
\hline Cardiac & $1(2.1 \%)$ & $3(4.8 \%)$ & 0.996 \\
\hline Gastrointestinal & $9(18.8 \%)$ & $12(19.0 \%)$ & 0.321 \\
\hline Hematologic & $4(8.3 \%)$ & $1(1.6 \%)$ & 0.164 \\
\hline Neurologic & $7(14.6 \%)$ & $14(22.2 \%)$ & 0.309 \\
\hline Oncology & $4(8.3 \%)$ & $9(14.3 \%)$ & 0.334 \\
\hline Pulmonary & $3(6.3 \%)$ & $3(4.8 \%)$ & 1.000 \\
\hline Renal & $1(2.1 \%)$ & $4(6.3 \%)$ & 0.387 \\
\hline Other & $5(10.4 \%)$ & $4(6.3 \%)$ & 0.497 \\
\hline None & $14(29.2 \%)$ & $13(20.6)$ & 0.299 \\
\hline
\end{tabular}




\section{Plasma Ang-1/2 at ED Presentation in Patients with Sepsis vs Septic Shock}

The median Ang-2 was higher in those with septic shock vs. those with sepsis [8,235 (IQR 4,712, $25,248) \mathrm{pg} / \mathrm{mL}$ vs. 5,659 (IQR 3,871, 7,808) pg/mL, $\mathrm{p}=0.008$ ). The quantile distribution is showed in Figure 1. The Ang-2/Ang-1 ratio was almost 2-fold greater in those with septic shock $[0.96(0.52,2.11)$ vs. 0.49 (IQR 0.33, 0.80), $\mathrm{p}=0.003$ ], (Table 2). Ang-1 was not statistically different in children with septic shock when compared to children with sepsis $(8,731$ (IQR $6,536,14,960) \mathrm{pg} / \mathrm{mL}$ vs. 11,883 (IQR 9,827, 17,680) $\mathrm{pg} / \mathrm{mL}, \mathrm{p}=0.075)$. Biomarker levels by group are shown in Table 2.

Table 2: Plasma Ang-1/2 values in sepsis vs. septic shock

\begin{tabular}{llll}
\hline Biomarker & Sepsis $(\mathrm{N}=45)$ & Septic Shock $(\mathrm{N}=49)$ & $\mathrm{p}$-value \\
\hline Ang-1 (median) & $11884 \mathrm{pg} / \mathrm{mL}$ & $8732 \mathrm{pg} / \mathrm{mL}$ & 0.075 \\
(IQR) & $(9827,17680)$ & $(6536,14960)$ & \\
Ang-2 (median) & $5659 \mathrm{pg} / \mathrm{mL}$ & $8235 \mathrm{pg} / \mathrm{ml}$ & 0.008 \\
(IQR) & $(3871,7808)$ & $(4712,25248)$ & \\
Ang2/ Ang1 Ratio & 0.49 & 0.96 & 0.003 \\
(IQR) & $(0.33,0.80)$ & $(0.52,2.11)$ & \\
\hline
\end{tabular}

Ang = Angiopoietin, $\mathrm{IQR}=$ interquartile range, $\mathrm{pg} / \mathrm{mL}=$ picograms per milliliter

\section{Plasma Ang-1/2 and Clinical Outcomes in Septic Shock}

There were no deaths in the study population. Among only the patients with septic shock, the initial Ang-2/Ang-1 ratio was associated with a higher dose of vasoactive agents at 12 hours, as calculated by the VIS $(\mathrm{p}=0.01)$, but not in total vasoactive time $(\mathrm{p}=0.14)$ (Table 3). In addition, a higher Ang-2/ Ang-1 ratio was associated with longer ICU LOS $(p=0.02)$, but not hospital LOS ( $\mathrm{p}=0.06)$. Neither Ang-2 nor Ang-1 alone was associated with maximum vasopressor score at 12 hours, total vasoactive time, ICU LOS, or hospital LOS. In addition, neither Ang-2/ Ang-1 ratio, Ang-2, nor Ang-1 alone were associated with degree of organ dysfunction at 24 hours as calculated using the PELOD score (Table 3).

In children with septic shock, for every 0.35 unit [95\% CI 0.18-0.51 $(\mathrm{p}<0.0009)]$ increase in the Ang-2/ Ang-1 ratio, the PRISM III score increased by 1. However, neither Ang-2 (0.00004, $\mathrm{P}=0.054)$ nor Ang-1 (-0.00011, $\mathrm{P}=0.263)$ alone correlated with PRISM III.

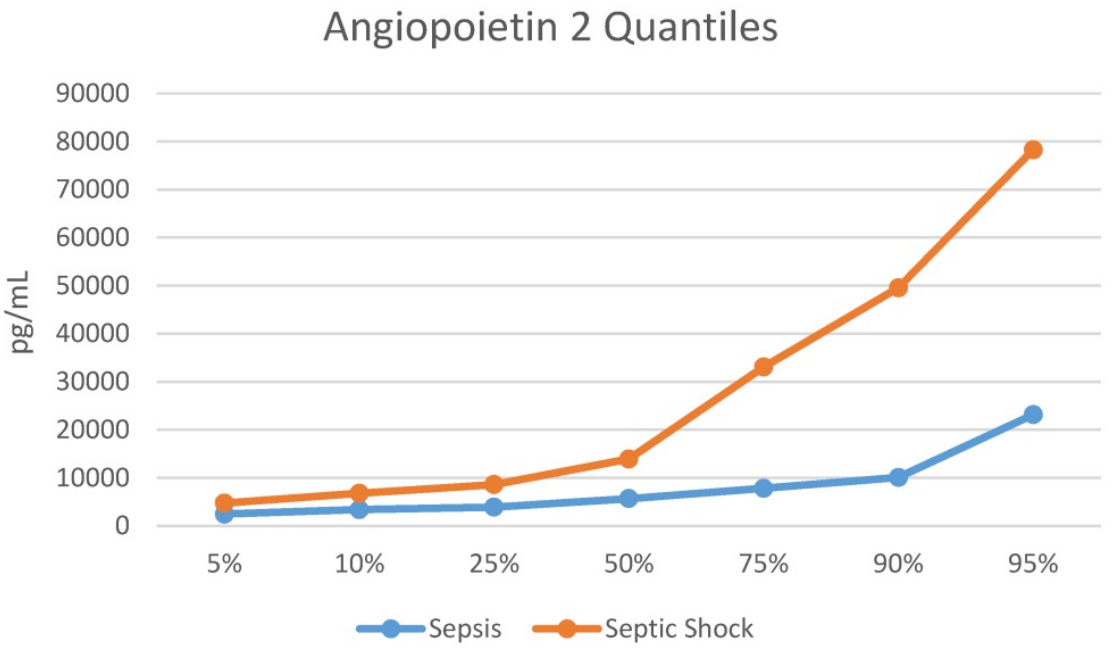

Figure 1: Quantile Distribution for Angiopoietin 2. $\mathrm{pg} / \mathrm{mL}=$ picograms $/$ milliliter

Table 3: Median regression analyses between plasma Ang-1, Ang-2, and Ang-2/Ang-1 ratio and clinical outcome measures among children with septic shock

\begin{tabular}{|c|c|c|c|c|c|}
\hline Biomarker & $\begin{array}{l}\text { ICU LOS in days }(95 \% \mathrm{CI}) \\
\text { p-value }\end{array}$ & $\begin{array}{l}\text { Vasoactive hrs: }(95 \% \mathrm{CI}) \\
\text { p-value }\end{array}$ & $\begin{array}{l}\text { Max 12hrs VIS: }(95 \% \mathrm{CI}) \\
\text { p-value }\end{array}$ & $\begin{array}{l}\text { Max PELOD 24hrs }(95 \% \mathrm{CI}) \\
\text { p-value }\end{array}$ & $\begin{array}{l}\text { PRISM-III: }(95 \% \mathrm{CI}) \\
\text { p-value }\end{array}$ \\
\hline Ang1 & $\begin{array}{l}(0.00,0.00) \\
p=0.60\end{array}$ & $\begin{array}{l}0.00(0.00,0.00) \\
P=0.57\end{array}$ & $\begin{array}{l}0.00(0.00,0.00) \\
p=0.72\end{array}$ & $\begin{array}{l}0.00(0.00,0.00) \\
p=1.00\end{array}$ & $\begin{array}{l}0.00(0.00,0.00) \\
p=0.26\end{array}$ \\
\hline Ang-2 & $\begin{array}{l}(0.00,0.00) \\
p=0.73\end{array}$ & $\begin{array}{l}0.00(0.00,0.00) \\
P=0.46\end{array}$ & $\begin{array}{l}0.00(0.00,0.00) \\
p=0.07\end{array}$ & $\begin{array}{l}0.00(0.00,0.00) \\
p=1.00\end{array}$ & $\begin{array}{l}0.00(0.00,0.00) \\
P=0.05\end{array}$ \\
\hline Ang-2/Ang-1 & $\begin{array}{l}0.14(0.02,0.26) \\
p=0.02\end{array}$ & $\begin{array}{l}0.00(-0.29,2.01) \\
P=0.14\end{array}$ & $\begin{array}{l}0.63(0.15,1.11) \\
p=0.01\end{array}$ & $\begin{array}{l}0.00(0.00,0.00) \\
p=1.00\end{array}$ & $\begin{array}{l}0.35(0.18,0.52) \\
\mathrm{p}<0.0009\end{array}$ \\
\hline
\end{tabular}

Ang $=$ Angiopoietin, $\mathrm{CI}=$ confidence interval, hrs $=$ hour, ICU LOS = Intensive Care Unit length of stay, PELOD = Pediatric Logistic Organ Dysfunction score, PRISM-III = Pediatric RISk of Mortality-III score, VIS = vasoactive inotrope score 


\section{Discussion}

This study shows that the Ang-2/Ang-1 ratio is associated with poor outcomes in children presenting to the emergency department with sepsis. In comparing children with septic shock to children with sepsis, Ang-2 and the Ang-2/Ang-1 ratio were statistically higher, showing that these biomarkers can be used to differentiate these two clinical scenarios on a biomarker basis. In those with septic shock specifically, a higher Ang-2/Ang-1 ratio is associated with a higher dose of vasoactive agents at 12 hours and a longer ICU length of stay.

Targeting biomarkers of endothelial activation and dysfunction in sepsis poses a unique opportunity to understand and perhaps modify the disease severity.4,22 Ang-2 promotes vascular injury and Ang-1 helps to maintain a healthy vascular system. Thus, in a state of septic shock where endothelial dysfunction predominates, Ang-2 should be elevated in those with a higher severity of illness and Ang-1 should be lower. As a result, greater separation of the Ang-2 and Ang-1 difference should be associated with worse severity of illness on presentation and worse outcomes as shown in this study.

Ang-2 has been shown to be elevated in adults with sepsis. ${ }^{23}$ The Ang-2/Ang-1 ratio was useful in stratifying risk of severe outcomes in adults with sepsis and correlated well with the severity of illness using the Mortality in Emergency Department Sepsis score. ${ }^{24}$ Mikacenic et al showed that higher Ang-2 levels were associated with worse clinical outcomes, while higher Ang-1 levels were associated with improved outcomes. ${ }^{11}$ Similarly, this was showed in children with severe bacterial infection. ${ }^{13}$ In children in the intensive care unit, Ang-2 levels were elevated in severe sepsis and septic shock. ${ }^{15,16}$ However, the relationship of Ang-1 and Ang-2 has not been previously studied in pediatric sepsis in the ED, a limitation of all the prior studies, where interventions could potentially have modified expression of Ang-1/2. This study specifically used plasma samples prior to any therapeutic intervention to determine if the initial ED sample was associated with clinical outcomes in pediatric sepsis.

Several validated scoring systems are in use to quantify pediatric ICU illness severity ${ }^{18}{ }^{25}$. Although an SOI scoring system exists for adults presenting to the emergency department with septic shock, ${ }^{24,26,27}$ a pediatric-specific sepsis score does not currently exist. Morin et al created a score for refractory septic shock but elements of the score include cardiac index, use of acute respiratory distress syndrome definition cutoffs, and the vasoactive inotrope score, values which are not available on entry to the ED ${ }^{28}$. Schlapback et al ${ }^{29}$ also developed a SOI score in pediatric sepsis, and though this functions better than PIM3, this score still uses data after 1 hour of admission to the pediatric ICU. As a result, none of the above SOI scores are useful at the point of entry to care. In the current study, the Ang-2/Ang-1 ratio correlated well with the eventual PRISM III score. This correlation may be useful in future studies by facilitating risk stratification prior to any therapeutic intervention. These findings have not been previously reported in studies of pediatric patients with sepsis.

Limitations of the study include the heterogeneous sample, including the difference of age between groups, the range of comorbidities at presentation, and presentation at various times in the course of illness. The impact of variable timing of presentation may have been mitigated by limiting enrollment to patients who met criteria less than twenty-four hours prior to ED presentation, and the requirement for a blood sample from our ED prior to any intervention; however, this limitation cannot be addressed completely. Measuring biomarkers in residual blood samples may have biased this data towards over-representation of sicker patients, since perhaps more blood was draw in those who were more ill. In addition, the patient's analyzed were a convenience sample cohort of patients who still had plasma available after a prior analysis, and thus the biomarker levels of excluded patients may have affected the observed results if these patients had been somewhat different than the final cohort included. Additionally, there is a possibility that thawing, and refreezing of the samples may have resulted in an unbalanced degradation rate based on handling. However, for these analyses, all samples were handled in a similar fashion with no bias to assignment. Finally, the performance of Angiopoietin was not compared to that of the white blood cell count, $\mathrm{C}$ - reactive protein, or procalcitonin, making the extrapolation that Angiopoietin is a better biomarker not possible.

Prediction of mortality was not feasible because there were none in the study population. This study may add to the understanding of clinical outcomes related to septic shock other than mortality, which may become increasingly important as care of patients with septic shock improves over time and mortality decreases. The lack of healthy control subjects for comparison is an additional limitation. Finally, the combination of treatments administered to children for septic shock was relatively standardized within our hospital; however, it was not proscriptive, and thus variation in treatment may be associated with differences in outcomes.

The results of the current study indicate that systemic Ang-1 and Ang-2 levels measured in plasma 
are clinically informative biomarkers of illness severity in sepsis that can be obtained from children at the time of first presentation to care. Elevated Ang-2/Ang-1 ratio values in ED samples were associated with higher doses of vasoactive agents at 12 hours and longer ICU length of stay, which may inform clinicians providing subsequent care. Future studies are warranted to better understand the prognostic utility of systemic Ang-1 and Ang-2 values as a child with sepsis progresses through its course, and how these values are affected by therapeutic interventions.

\section{Conclusions}

The Ang-2/ Ang-1 ratio is higher in children with septic shock compared to children with sepsis without septic shock. Ang-2/Ang-1 when measured in the those specifically with septic shock is associated with higher requirement of vasoactive agents at 12 hours and longer ICU length of stay, and the initial level correlates with the ICU severity score obtained later in the patients' course.

\section{Competing Interests}

Dr. Liles is listed as an inventor on US patent, "Biomarkers for Early Determination of a Critical or Life-Threatening Response to Illness and/or Treatment Response." The remaining authors have no financial relationships relevant to this article to disclose.

\section{References}

1. Watson RS, Carcillo JA, Linde-Zwirble WT, Clermont G, Lidicker J, Angus DC. The epidemiology of severe sepsis in children in the United States. Am J Respir Crit Care Med 2003;167:695-701.

2. Hartman ME, Linde-Zwirble WT, Angus DC, Watson RS. Trends in the epidemiology of pediatric severe sepsis*. Pediatr Crit Care Med 2013;14:686-93.

3. Aird WC. The role of the endothelium in severe sepsis and multiple organ dysfunction syndrome. Blood 2003;101:3765-77.

4. Page AV, Liles WC. Biomarkers of endothelial activation/dysfunction in infectious diseases. Virulence 2013;4:507-16.

5. Pierrakos C, Vincent JL. Sepsis biomarkers: a review. Crit Care 2010;14:R15

6. Fiedler U, Augustin HG. Angiopoietins: a link between angiogenesis and inflammation. Trends Immunol 2006;27:552-8.

7. Thurston G, Rudge JS, Ioffe E, et al. Angiopoietin-1 protects the adult vasculature against plasma leakage. Nat Med 2000;6:460-3.

8. Witzenbichler B, Westermann D, Knueppel S, Schultheiss HP, Tschope C. Protective role of angiopoietin-1 in endotoxic shock. Circulation 2005;111:97-105

9. Fiedler U, Reiss $Y$, Scharpfenecker M, et al. Angiopoietin-2 sensitizes endothelial cells to TNF-alpha and has a crucial role in the induction of inflammation. Nat Med 2006;12:235-9.

10. Parikh SM, Mammoto T, Schultz A, et al. Excess circulating angiopoietin-2 may contribute to pulmonary vascular leak in sepsis in humans. PLoS Med 2006:3:e46.

11. Mikacenic C, Hahn WO, Price BL, et al. Biomarkers of Endothelial Activation Are Associated with Poor Outcome in Critical Illness. PLoS One 2015;10:e141251.

12. Ricciuto DR, dos Santos CC, Hawkes M, et al. Angiopoietin-1 and angiopoietin-2 as clinically informative prognostic biomarkers of morbidity and mortality in severe sepsis. Crit Care Med 2011;39:702-10.

13. Mankhambo LA, Banda DL, Group IPDS, et al. The role of angiogenic factors in predicting clinical outcome in severe bacterial infection in Malawian children. Crit Care 2010;14:R91.
14. Lovegrove FE, Tangpukdee N, Opoka RO, et al. Serum angiopoietin-1 and -2 levels discriminate cerebral malaria from uncomplicated malaria and predict clinical outcome in African children. PLoS One 2009;4:e4912.

15. Giuliano JS, Jr., Tran K, Li FY, Shabanova V, Tala JA, Bhandari V. The temporal kinetics of circulating angiopoietin levels in children with sepsis. Pediatr Crit Care Med 2014;15:e1-8.

16. Giuliano JS, Jr., Lahni PM, Harmon K, et al. Admission angiopoietin levels in children with septic shock. Shock 2007;28:650-4.

17. Goldstein B, Giroir B, Randolph A, International Consensus Conference on Pediatric S. International pediatric sepsis consensus conference: definitions for sepsis and organ dysfunction in pediatrics. Pediatr Crit Care Med 2005;6:2-8.

18. Pollack MM, Patel KM, Ruttimann UE. The Pediatric Risk of Mortality III--Acute Physiology Score (PRISM III-APS): a method of assessing physiologic instability for pediatric intensive care unit patients. J Pediatr 1997;131:575-81.

19. Leteurtre S, Martinot A, Duhamel A, et al. Validation of the paediatric logistic organ dysfunction (PELOD) score: prospective, observational, multicentre study. Lancet 2003;362:192-7.

20. Gaies MG, Gurney JG, Yen AH, et al. Vasoactive-inotropic score as a predictor of morbidity and mortality in infants after cardiopulmonary bypass. Pediatr Crit Care Med 2010;11:234-8.

21. Harris PA, Taylor R, Thielke R, Payne J, Gonzalez N, Conde JG. Research electronic data capture (REDCap)--a metadata-driven methodology and workflow process for providing translational research informatics support. J Biomed Inform 2009;42:377-81.

22. Xing K, Murthy S, Liles WC, Singh JM. Clinical utility of biomarkers of endothelial activation in sepsis--a systematic review. Crit Care 2012;16:R7.

23. Orfanos SE, Kotanidou A, Glynos C, et al. Angiopoietin-2 is increased in severe sepsis: correlation with inflammatory mediators. Crit Care Med 2007;35:199-206.

24. Jones AE, Saak K, Kline JA. Performance of the Mortality in Emergency Department Sepsis score for predicting hospital mortality among patients with severe sepsis and septic shock. Am J Emerg Med 2008;26:689-92.

25. Wolfler A, Osello R, Gualino J, et al. The Importance of Mortality Risk Assessment: Validation of the Pediatric Index of Mortality 3 Score. Pediatr Crit Care Med 2016;17:251-6.

26. Carpenter CR, Keim SM, Upadhye S, Nguyen HB, Best Evidence in Emergency Medicine Investigator G. Risk stratification of the potentially septic patient in the emergency department: the Mortality in the Emergency Department Sepsis (MEDS) score. J Emerg Med 2009;37:319-27.

27. Macdonald SP, Arendts G, Fatovich DM, Brown SG. Comparison of PIRO, SOFA, and MEDS scores for predicting mortality in emergency department patients with severe sepsis and septic shock. Acad Emerg Med 2014;21:1257-63.

28. Morin L, Ray S, Wilson C, et al. Refractory septic shock in children: a European Society of Paediatric and Neonatal Intensive Care definition. Intensive Care Med 2016;42:1948-57.

29. Schlapbach LJ, MacLaren G, Festa M, et al. Prediction of pediatric sepsis mortality within $1 \mathrm{~h}$ of intensive care admission. Intensive Care Med 2017;43:1085-96. 